\title{
WSN Based on Bio-Inspired Algorithm in Cluster Head Formation
}

\author{
Vinmathi $\mathrm{M} \mathrm{S}^{\mathrm{a}, 1}$, Josephine $\mathrm{M} \mathrm{S}^{\mathrm{b}}$, and Jeyabalaraja $\mathrm{V}^{\mathrm{c}}$ \\ ${ }^{a}$ Research scholar-CSE, ${ }^{b}$ Professor-Computer Applications \\ ${ }^{a, b}$ Dr.M.G.R. Educational and Research Institute, Chennai \\ 'Professor, Dept of CSE, Velammal Engineering College, Chennai
}

\begin{abstract}
A sort of inclusion improvement technique dependent on bio-inspired algorithm was implemented so as to fathom the irrationality and low system inclusion of sensor hub in WSN at the irregular dispersion. Right off the bat, the ebb and flow investigate status of WSN inclusion was broke down, the hub inclusion and territorial inclusion in WSN on the premise were examined, the comparing scientific model was set up, the bio-enlivened calculation was taken to tackle the built up numerical model, and the WSN inclusion advancement program dependent on the bio-roused was gotten. At last, MATLAB was utilized for the recreation analyses, and the reproduction results demonstrated that the presentation of bio-motivated calculation improved the hub inclusion in WSN viably; the inclusion territory was huger at a similar measure of hubs. In addition, the calculation can get the ideal arrangement in the worldwide extension, and arrive at the better system inclusion advancement impact with less sensor hubs, and the quantity of cycles was diminished altogether.
\end{abstract}

Keywords: Wireless Sensor Networks (WSN); bio-inspired method; sensor node

\section{Introduction}

Remote sensor systems have as of late achieved the aggregate consideration similarly from the examination network and real clients. A few grouping calculations in regards to various settings have just been proposed previously. Among those calculations numerous calculations are especially center around lessening the vitality devoured in the institutionalized framework. For the most part, directing ends up being an extra testing and evaluative, when the size of the system scales up. As of late, the organically propelled scholarly calculations have been built up so as to manage this specific issue. The product specialists can be shaped to disentangle complex issues, by utilizing ants, honey bees and other social swarms as models, for example, traffic re-directing in occupied media transmission systems. It blended the clamorous movement and molecule swarm calculation. It conquered the moderate settling speed, while it was anything but difficult to fall in the neighbourhood outrageous worth [1].In the interim, the multifaceted nature of the calculation was higher, and the unravelling procedure was mind boggling. Writing [2] a half breed hereditary calculation, the specific

\footnotetext{
${ }^{1}$ Vinmathi M S, research scholar-CSE, Dr.M.G.R. Educational and Research Institute, Chennai Email: vinmathis@gmail.com
} 
arrangement can be unravelled quickly, while the vitality of system hub was not considered, and the estimation was mind boggling generally, and it was not helpful for sensor hub with constrained vitality. A sort of WSN inclusion enhancement technique dependent on improved bio-roused calculation was included in the paper to improve the WSN inclusion.

\section{WSN coverage based on bio-inspired algorithm}

\subsection{Bio-inspired algorithm}

There are sure issues in WSNs that have been communicated as multidimensional streamlining issues, and are settled by using bio-enlivened techniques. There are likewise some extra issues that are every now and again communicated as advancement issues, for example, hub position, information assortment, limitation, and vitality based clustering. As sensor systems scale up in size, the administration with respect to circulation of the systems administration load successfully will be the most profound worry of the sensor hubs. Burden adjusting midpoints the utilization of vitality in a successful manner by dispersing the outstanding task at hand among the groups in sensor arrange. Burden adjusting is likewise helpful so as to limit the blockage problem areas, which thus prompts the decrease of remote impacts.

\subsection{Foraging behavior}

$$
X_{j}=X_{i}+\text { visual.Rand }()
$$

If the food concentration $Y=f(X)$ at the current location of bio-inspired meets $Y_{i}<Y_{j}$ in the solution of maximum problem, the direction moves towards for one step.

$$
X_{i}^{t+1}=X_{i}^{t}+\frac{X_{j}-X^{t}}{\left\|X_{j}-X_{i}^{t}\right\|} \bullet \text { step } \bullet \operatorname{Rand}()
$$

In the formula, Step is the moving step length. Otherwise, the state $X_{j}$ is selected randomly again, whether meeting the forward condition is judged, the repeated attempt is conducted. After tried for Try_number, if the forward condition cannot be met, one step is moved randomly within the field of vision

$$
X_{i}^{t+1}=X_{i}^{t}+\text { visual.Rand }()
$$




\subsection{Bunching behavior}

The fish would bunch in the swimming process in the natural field to guarantee the group survival and elude the danger. Two rules are prescribed for the bunching behavior of each bio-inspired among the bio-inspired algorithm: firstly is trying to move towards the center of neighboring partner; and secondly is to avoid the overcrowding.

$$
X_{i}^{t+1}=X_{i}^{t}+\frac{X_{c}-X^{t}}{\left\|X_{c}-X_{i}^{t}\right\|} \bullet \text { step } \bullet \operatorname{Rand}()
$$

Otherwise, the foraging behavior shall be implemented.

\subsection{Random behavior}

The random behavior can be regarded as the default behavior in foraging behavior. It can be described as the free swimming of fish in the water simply. Although it is random, the purpose is to seek the food or partner within a broader range, which means that the fish can select a state randomly in the vision, and then move towards the direction.

\section{WSN Explanation}

WSN applications can be delegated Tracking and Monitoring. Following incorporates foe following, Human or Traffic following and checking includes security discovery in Military, creature observing, business checking, Industrial checking, Patient observing and Environmental observing [3]. WSNs have fantastic research enthusiasm because of their on-going applications in Civil and Military field. Social event of sensor information, transmission and controlling relying upon the applications is in-worked in these frameworks. The primary target of this paper is to exhibit a diagram of WSN Optimization utilizing various developmental models and to examine the current papers identified with this field. A Design challenges for WSN There are a few highlights of WSNs that [distinguish them from customary remote impromptu systems. To begin with, WSNs have explicit information stream designs as multicast (one-to-many) and combine cast (numerous to-one) trees [4].

Second, WSNs are normally comprised of various little hubs furnished with low end processors, constrained non-battery-powered battery, little data transfer capacity connections and little memory. WSN convention creators face severe imperatives on the utilization and the accessibility of sensor hub assets [5]. Third, most of uses for Wireless Sensor Networks require the arrangement of the hubs in gigantic sum, going from thousands to millions. Consequently, the versatility of the pre-owned conventions is likewise a significant issue [6]. Fourth, singular sensor hubs would themselves be able to create enormous measures of information. The information transmission from the sensor hubs to a typical sink hub would devour an enormous measure of vitality, 
transfer speed, and force. This issue can be settled by distinguishing and sifting the excess data to decrease the system traffic. There are some particular qualities of WSNs that the directing conventions must have so as to permit their utilization in true applications.

\section{Interpretation of Results}

\subsection{Experimental setup}

"Inclusion" is one of the most significant presentation measurements for sensors to organize. In different remote correspondence systems, inclusion ordinarily implies the radio inclusion. On the other hand, inclusion with regards to WSNs relates to the detecting range, while availability compares to the correspondence go. WSN inclusion can be grouped into three sorts: zone inclusion, point inclusion and boundary inclusion [7]. In region inclusion, the inclusion nature of a whole two-dimensional (2D) district is considered, where each point in the area is seen by in any event one sensor hub. In point inclusion, the goal is to just ensure that a limited arrangement of focuses in the area is seen by in any event one sensor hub. Obstruction inclusion for the most part manages the recognition of development over a boundary of sensor hubs. The most luxuriously considered inclusion issue in the WSN writing is the region inclusion issue.

Another issue in WSN configuration is the system network that is reliant on the chosen correspondence convention [8]. Two sensor hubs are legitimately associated if the separation of the two hubs is littler than the correspondence extend Rc. Availability just necessitates that the area of any dynamic hub is inside the correspondence scope of at least one dynamic hubs, with the goal that every single dynamic hub can frame an associated correspondence organize [9]. The most well-known convention depends on a bunch based design, where all hubs in a similar group can legitimately speak with one another by means of a solitary jump and all hubs in a similar group can speak with all hubs in the neighbouring bunches through the bunch head. In a given group just a solitary hub goes about as the bunch head, which must be dynamic as far as gathering all data gathered by different hubs for looking after availability. In bunch based WSNs, the network issues will in general rely on the quantity of hubs in each group

Another significant exhibition metric in WSNs is their lifetime. Gigantic research endeavors have been put into taking care of the issue of delaying system lifetime by vitality preservation in WSNs. In fact, the vitality wellspring of every hub is commonly restricted, while reviving or supplanting the battery at the sensors might be incomprehensible. Thus, both the radio handset unit and the sensor unit of every hub must be vitality productive, and it is essentially critical to amplify the achievable system lifetime. 


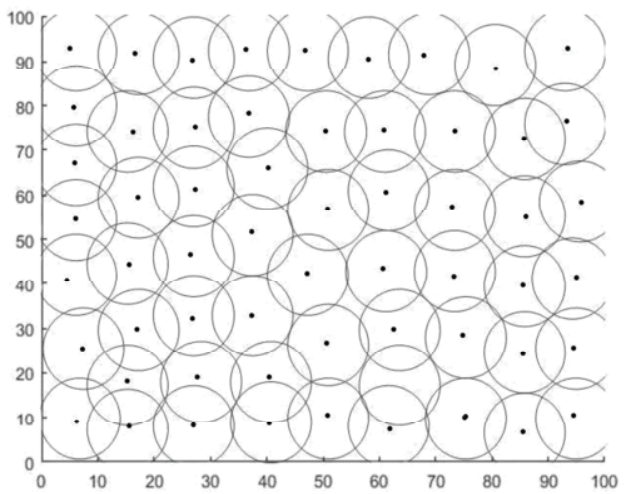

Figure 1. Arbitrarily sensor hub circulation

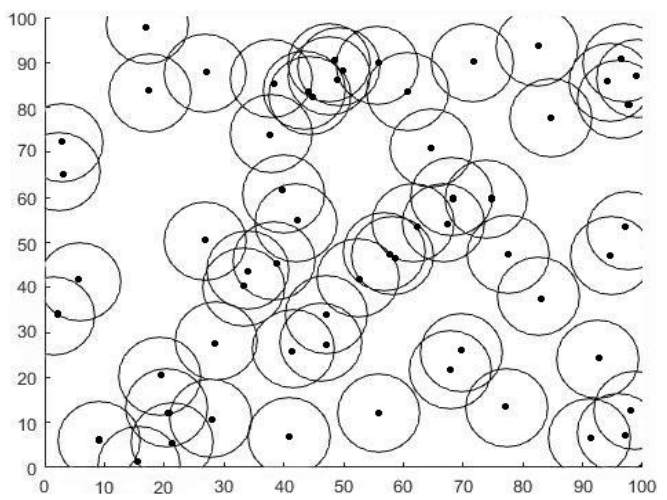

Figure 2. Dissemination of Sensor Nodes

\section{Conclusion}

Commonly, sensor hubs are gathered into groups, and each bunch has a hub that goes about as the group head, which has a bigger number of assets and computational force than the other group hubs. All hubs accumulate and convey their detected data to the bunch head, which thusly advances it to a particular hub, in particular the sink hub or base station, by means of a bounce by-jump remote correspondence interface. In indoor situations, a WSN is normally rather little and comprises of a solitary group bolstered by a solitary base station. Numerous bunches depending on different base stations are conceivable in a huge scope organization of WSNs.

\section{References}

[1] Akyildiz I F, Su Wei-Lian, Sankarasubramaniam Y, et al. A survey on sensor networks. IEEE Communications Magazine, 2002,40(8) . 102-114.

[2] JINAG Ming-yan, YUAN Dong-feng. Artificial fish school algorithm and application. BeiJing: Sciens Press, 2012. 261-269.

[3] REN Yan, ZHANG Si-Dong, and ZHANG Hong-Ke. Theories and algorithms of coverage control for wireless sensor networks. Journal of Software, 2006,17(3) . 422-433. 
[4] Wang Xue-Qing, YANG Yong-Tian, SUN Ting, et al. Research on the gird based coverage problem in wireless sensor networks. Computer Science,2006,33(11) . 38-39,78.

[5] ZENG Ying-lan, CHEN Jing, ZHENG Jin-hua. Genetic algorithm-based sensor network coverageenhancing approach. Computer Engineering and Applications,2009,45(11) . 89-91.

[6] HUANG Yu-yue, LI Ke-qing. Coverage optimization of wireless sensor networks based on artificial fish swarm algorithm. Application Research of Computers,2013,30(2) . 554-556.

[7] LIU Wei-ting, FAN Zhou-yuan. Coverage optimization of wireless sensor networks based on chaos particle swarm algorithm. Journal of Computer Applications,2011,31(2) . 338-340,361.

[8] LIN Mei-jin, SU Cai-hong, WANG Fei. Study on optimization algorithms in wireless sensor networks. Computer Simulation, 2011,28(3) .178-181.

[9] Shaofei Wu,A Traffic Motion Object Extraction Algorithm,International Journal of Bifurcation and Chaos,2015,25(14).Article Number 1540039. 